\title{
RELAXATION AND ATTAINMENT RESULTS FOR AN INTEGRAL FUNCTIONAL WITH UNBOUNDED ENERGY-WELL
}

\author{
BAISHENG YAN
}

\begin{abstract}
Consider functional $I(u)=\left.\int_{\Omega}|| D u\right|^{n}-L \operatorname{det} D u \mid d x$, whose energy-well consists of matrices satisfying $|\xi|^{n}=L \operatorname{det} \xi$. We show that the relaxations of this functional in various Sobolev spaces are significantly different. We also make several remarks concerning various $p$-growth semiconvex hulls of the energy-well set and prove an attainment result for a special Hamilton-Jacobi equation, $|D u|^{n}=L \operatorname{det} D u$, in the so-called grand Sobolev space $W^{1, q)}\left(\Omega ; \mathbf{R}^{n}\right)$ with $q=\frac{n L}{L+1}$.
\end{abstract}

\section{IntRoduction AND MAIN RESUlts}

Given $L \geq 1$, consider function $f_{L}(\xi)=|\xi|^{n}-L \operatorname{det} \xi$ on the space $\mathbb{M}^{n \times n}$ of $n \times n$ matrices and integral functional

$$
I(u)=\int_{\Omega}\left|f_{L}(D u(x))\right| d x=\left.\int_{\Omega}|| D u(x)\right|^{n}-L \operatorname{det} D u(x) \mid d x,
$$

where $u$ is a mapping from domain $\Omega \subset \mathbf{R}^{n}$ to $\mathbf{R}^{n}$ and $D u(x)$ is the Jacobi matrix of $u$. In general, $|\xi|$ denotes the operator norm of $m \times n$ matrix $\xi \in \mathbb{M}^{m \times n}$ defined by $|\xi|=\max _{h \in \mathbf{R}^{n},|h|=1}|\xi h|$.

The absolute energy minimizers of $I(u)$ can be characterized as mappings satisfying the Hamilton-Jacobi equation:

$$
D u(x) \in Z_{L}=\left\{\left.\xi \in \mathbb{M}^{n \times n}|| \xi\right|^{n}=L \operatorname{det} \xi\right\} \quad \text { a.e. } x \in \Omega \text {. }
$$

In the terminology used for phase transition problems (see, e.g., [3, 9, $10,11]$ ), the set $Z_{L}$ is the energy-well of energy functional $I(u)$. Note that $Z_{L}$ is also the boundary of the so-called $L$-quasiconformal set $K_{L}$ defined by

$$
K_{L}=\left\{\left.\xi \in \mathbb{M}^{n \times n}|| \xi\right|^{n} \leq L \operatorname{det} \xi\right\}
$$


When $L=1$, it is easily seen that the set $K_{1}=Z_{1}$ coincides with the set of conformal matrices, that is,

$$
K_{1}=Z_{1}=\{\lambda R \mid \lambda \geq 0, R \in S O(n)\} .
$$

Weakly $L$-quasiregular mappings are the mappings $u \in W_{l o c}^{1, p}\left(\Omega ; \mathbf{R}^{n}\right)$ satisfying $D u(x) \in K_{L}$ almost everywhere in $\Omega$ (see Iwaniec [6]). Many regularity and stability properties of weakly quasiregular mappings have been studied in connection with the certain semi-convex hulls and the attainment result of the quasiconformal sets $K_{L}$ in Yan $[13,14,15]$. In this paper we study similar problems related to the set $Z_{L}$.

The natural space for $I(u)$ is the Sobolev space $W^{1, n}\left(\Omega ; \mathbf{R}^{n}\right)$. In this note we are interested in minimization of functional $I(u)$ in different Sobolev spaces $W^{1, p}\left(\Omega ; \mathbf{R}^{n}\right)$. For this purpose, we define the $p$-growth relaxation functions of $I(u)$ as follows:

$$
g_{p}(\xi)=\inf _{u \in W^{1, p}\left(\Omega ; \mathbf{R}^{n}\right),\left.u\right|_{\partial \Omega}=\xi x} \frac{1}{|\Omega|} \int_{\Omega}\left|f_{L}(D u(x))\right| d x .
$$

Clearly the function $g_{p}(\xi)$ is non-decreasing with respect to $p \geq 1$. For any given function $f: \mathbb{M}^{m \times n} \rightarrow \mathbf{R}$, we define the quasiconvexification of $f$ to be

$$
f^{\#}(\xi)=\inf _{\phi \in C_{0}^{\infty}\left(\Omega ; \mathbf{R}^{m}\right)} \frac{1}{|\Omega|} \int_{\Omega} f(\xi+D \phi(x)) d x, \quad \forall \xi \in \mathbb{M}^{m \times n},
$$

where $C_{0}^{\infty}\left(\Omega ; \mathbf{R}^{m}\right)$ stands for all smooth functions with compact support in $\Omega$. A density argument easily shows that $g_{\infty}=\left|f_{L}\right|^{\#}$ and hence the function $g_{\infty}$ is also quasiconvex in the sense of Morrey. Recall that, in general, a function $f: \mathbb{M}^{m \times n} \rightarrow \mathbf{R}$ is said to be quasiconvex (in the sense of Morrey) if it satisfies

$$
\frac{1}{|\Omega|} \int_{\Omega} f(\xi+D \phi(x)) d x \geq f(\xi), \quad \forall \xi \in \mathbb{M}^{m \times n}, \phi \in C_{0}^{\infty}\left(\Omega ; \mathbf{R}^{m}\right) .
$$

It is well-known that any finite quasiconvex function $f$ is always rankone convex; that is, $f(\xi+t \eta)$ is convex in $t \in \mathbf{R}$ for any $\xi, \eta \in \mathbb{M}^{m \times n}$ with $\operatorname{rank} \eta=1$. A function $f: \mathbb{M}^{m \times n} \rightarrow \mathbf{R}$ is called polyconvex if it can be written as a convex function of all subdeterminants; therefore all convex functions are polyconvex. It can be shown that a polyconvex function is always quasiconvex. We refer to Acerbi \& Fusco [1], Ball [2], Ball \& Murat [4], Dacorogna [5], Morrey [8], and Müller [9] for the proofs and more properties of these semiconvex functions. 
In what follows, we denote by $f^{-1}(0)$ the zero set of any scalar function $f$, that is, $f^{-1}(0)=\{\xi \mid f(\xi)=0\}$. We now state our main result.

Theorem 1.1. Let $n \geq 3$ and $L \geq 1$. Then

(a) $g_{p}=\left|f_{L}\right|^{\#}=\max \left\{f_{L}, 0\right\}$ for all $p \geq n$;

(b) for some $\epsilon>0, g_{p}^{-1}(0)=K_{L}$ for all $p \geq n-\epsilon$;

(c) $g_{p} \equiv 0$ and the minimum is attained for all $1 \leq p<\frac{n L}{L+1}$.

Part (c) of the theorem is equivalent to the following

Theorem 1.2. Let $n \geq 3, L \geq 1$. Then, for any $1 \leq p<\frac{n L}{L+1}$ and $\xi \in$ $\mathbb{M}^{n \times n}$, there exists a map $u \in W^{1, p}\left(\Omega ; \mathbf{R}^{n}\right)$ with $\left.u\right|_{\partial \Omega}=\xi x$ satisfying

$$
|D u(x)|^{n}=L \operatorname{det} D u(x) \quad \text { a.e. } x \in \Omega \text {. }
$$

Remark. This result sharpens an earlier result in Yan [14]. Note that, if $\xi=0$, the trivial map $u \equiv 0$ is a required solution of (1.6); but, in this case, we can show existence of nontrivial solutions. In fact, we shall prove a sharper attainment result for a special class of nontrivial solutions of (1.6) in a space strictly smaller than any $W^{1, p}\left(\Omega ; \mathbf{R}^{n}\right)$ for $1 \leq p<\frac{n L}{L+1}$.

For a given $q>1$, we define the grand Sobolev space $W^{1, q)}\left(\Omega ; \mathbf{R}^{m}\right)$ (see e.g. Iwaniec \& Sbordone [7]) to be the space of all functions $u \in \cap_{1 \leq p<q} W^{1, p}\left(\Omega ; \mathbf{R}^{m}\right)$ that satisfy

$$
[D u]_{q, \Omega} \equiv \sup _{1 \leq p<q}\left[(q-p) \frac{1}{|\Omega|} \int_{\Omega}|D u(x)|^{p} d x\right]^{1 / p}<\infty .
$$

Given any number $\alpha \in \mathbf{R}$, we define the set

$$
S_{\alpha}=\left\{R(I+\alpha \omega \otimes \omega)\left|R \in Z_{1}, R \neq 0, \omega \in \mathbf{R}^{n},\right| \omega \mid=1\right\},
$$

where $I$ is $n \times n$ identity matrix and $a \otimes b$ stands for the rank-one matrix $\left(a_{i} b_{j}\right)$. In the following, we shall use $\delta\left(\epsilon_{1}, \epsilon_{2}, \cdots, \epsilon_{n}\right)$ to denote the $n \times n$ diagonal matrix with diagonal elements $\epsilon_{1}, \ldots, \epsilon_{n}$; that is, we denote

$$
\delta\left(\epsilon_{1}, \epsilon_{2}, \cdots, \epsilon_{n}\right)=\left(\begin{array}{llll}
\epsilon_{1} & & & 0 \\
& \epsilon_{2} & & \\
& & \ddots & \\
0 & & & \epsilon_{n}
\end{array}\right) .
$$

Let $J=I-2 e_{1} \otimes e_{1}=\delta(-1,1, \cdots, 1)$. Then $J^{2}=I$ and $\operatorname{det} J=-1$. Define $J S_{\alpha}=\left\{J \xi \mid \xi \in S_{\alpha}\right\}$. Note that any matrix $\xi \in S_{\alpha}$ can be 
written as $\xi=\lambda Q(I+\alpha \omega \otimes \omega)$ for some $\lambda>0, Q \in S O(n)$ and $\omega \in \mathbf{R}^{n}$ with $|\omega|=1$. Therefore, it follows easily that $\operatorname{det} \xi=\lambda^{n}(1+\alpha)$ and $|\xi|=\lambda$ if $|1+\alpha| \leq 1$ or $|\xi|=\lambda|1+\alpha|$ if $|1+\alpha| \geq 1$. From this, one easily proves the following

Proposition 1.3. For $\alpha=L^{\frac{1}{n-1}}-1$ or $L^{-1}-1, S_{\alpha} \subset Z_{L}$; for $\alpha=$ $-L^{\frac{1}{n-1}}-1$ or $-L^{-1}-1, J S_{\alpha} \subset Z_{L}$.

In this paper, we shall also prove the following much stronger attainment result.

Theorem 1.4. Let $n \geq 3, L \geq 1$ be given. Then, for any $\xi \in \mathbb{M}^{n \times n}$, there exists a map $u$ in the grand Sobolev space $W^{\left.1, \frac{n L}{L+1}\right)}\left(\Omega ; \mathbf{R}^{n}\right)$ such that

$$
\left.u\right|_{\partial \Omega}=\xi x, \quad D u(x) \in S_{\alpha_{1}} \cup J S_{\alpha_{2}} \subset Z_{L} \quad \text { a.e. } x \in \Omega,
$$

for $\alpha_{1}=L^{\frac{1}{n-1}}-1$ or $L^{-1}-1$ and $\alpha_{2}=-L^{-1}-1$, where the boundary condition $\left.u\right|_{\partial \Omega}=\xi x$ is satisfied in the $W^{1, p}$-sense for all $1 \leq p<\frac{n L}{L+1}$.

Given any subset $K$ of $\mathbb{M}^{m \times n}$ and a number $p \geq 1$, let $C_{p}^{+}(K)$ be the set of continuous functions $f: \mathbb{M}^{m \times n} \rightarrow \mathbf{R}$ satisfying

$$
0 \leq f(\xi) \leq C\left(|\xi|^{p}+1\right),\left.\quad f\right|_{K}=0 .
$$

Let $Q C_{p}^{+}(K), R C_{p}^{+}(K)$ and $P C_{p}^{+}(K)$ be the class of quasiconvex, rankone convex, and polyconvex functions in $C_{p}^{+}(K)$, respectively.

Definition 1.1. The p-growth quasiconvex, rank-one convex and polyconvex hulls of set $K$ are defined, respectively, as follows:

$$
\begin{aligned}
& Q_{p}(K)=\cap\left\{f^{-1}(0) \mid f \in Q C_{p}^{+}(K)\right\} ; \\
& R_{p}(K)=\cap\left\{f^{-1}(0) \mid f \in R C_{p}^{+}(K)\right\} ; \\
& P_{p}(K)=\cap\left\{f^{-1}(0) \mid f \in P C_{p}^{+}(K)\right\} .
\end{aligned}
$$

Remark. These $p$-growth semiconvex hulls have been referred to as the $W^{1, p}$ - or, simply, $p$-semiconvex hulls in Yan $[12,15]$ and Yan \& Zhou [16, 17], following the definition of usual semiconvex hulls without growth restriction given, for example, in Müller \& Šverák [10] and Šverák [11]. We adopt the present definition here to stress the growth condition and to distinguish with the different and not equivalent definition of $p$-semiconvex hulls given in Zhang $[18,19]$.

The $p$-growth semiconvex hulls of the set $K_{L}$ have been studied in Yan [15]. Concerning the set $Z_{L}$, we have the following 
Theorem 1.5. A p-growth semiconvex hull of $Z_{L}$ is the same as that of $K_{L}$. For example, $R_{p}\left(Z_{L}\right)=\mathbb{M}^{n \times n}$ for $p<\frac{n L}{L+1}$ and $R_{p}\left(Z_{L}\right)=K_{L}$ for $p \geq \frac{n L}{L+1}$; moreover, $Q_{p}\left(Z_{L}\right)=K_{L}$ for $p \geq n-\epsilon$ with some $\epsilon>0$.

\section{Relaxations AND SEMiCONVEX HULLS}

In this section, we prove Theorem 1.1, parts (a) and (b), and Theorem 1.5. Part (c) of Theorem 1.1 and Theorem 1.2 follow from Theorem 1.4 , which will be proved later in the paper.

First of all, we observe the following useful result.

Lemma 2.1. $\lim _{|t| \rightarrow \infty} f_{L}(\xi+t \eta)=+\infty$ for any given $\xi, \eta \in \mathbb{M}^{n \times n}$ with $\operatorname{rank} \eta=1$.

Proof. It suffices to note that $\operatorname{det}(\xi+t \eta)=\operatorname{det} \xi+c t$ for a constant $c$ if $\operatorname{rank} \eta=1$, and hence

$$
f_{L}(\xi+t \eta) \geq(|t||\eta|-|\xi|)^{n}-|c||t|-|\operatorname{det} \xi| \rightarrow \infty
$$

as $|t| \rightarrow \infty$.

Proof of Theorem 1.1(a). Using a well-known relaxation principle for integral functionals (see, e.g., $[1,5]$ ) one can easily show that $g_{n}=$ $\left|f_{L}\right|^{\#}$ and hence, by definition,

$$
0 \leq g_{p} \leq g_{q} \leq\left|f_{L}\right|^{\#} \leq\left|f_{L}\right|, \quad \forall p \leq q
$$

Since $f_{L}=|\xi|^{n}-L \operatorname{det} \xi$ is $W^{1, n}$-quasiconvex [4], we easily have $g_{n} \geq f_{L}$ and hence $g_{n} \geq \max \left\{f_{L}, 0\right\}$. (This can be also derived directly from property of the determinant.) Therefore, by (2.1), to prove Theorem 1.1(a), it is sufficient to show

$$
\left|f_{L}\right|^{\#}=\max \left\{f_{L}, 0\right\} .
$$

But this follows from Lemma 2.1 and the following general result:

Proposition 2.2. Let $f$ be a quasiconvex function. Suppose that for each $\xi \in \mathbb{M}^{n \times n}$ there exists a rank-one matrix $\eta$ such that

$$
\lim _{|t| \rightarrow \infty} f(\xi+t \eta)=+\infty
$$

Then $|f|^{\#}=\max \{f, 0\}$. 
Proof. Obviously, since $f$ is quasiconvex, $|f|^{\#} \geq \max \{f, 0\}$. We prove the equality. Suppose, for the contrary, $|f|^{\#}\left(\xi_{0}\right)>\max \left\{f\left(\xi_{0}\right), 0\right\}$ for some $\xi_{0}$. Since $|f|^{\#}\left(\xi_{0}\right) \leq\left|f\left(\xi_{0}\right)\right|$ we must have $f\left(\xi_{0}\right)<0$. Now consider $h(t)=f\left(\xi_{0}+t \eta_{0}\right)$ where $\eta_{0}$ is a rank-one matrix such that $h(t) \rightarrow+\infty$ as $|t| \rightarrow \infty$. Then, since $h(0)=f\left(\xi_{0}\right)<0$, we would have $t_{1}<0<t_{2}$ such that $h\left(t_{1}\right)=h\left(t_{2}\right)=0$. This implies $|f|^{\#}\left(\xi_{0}+t_{i} \eta_{0}\right)=0$ for $i=1,2$. From this, since $|f|^{\#}$ is rank-one convex, we would arrive at the conclusion that $|f|^{\#}\left(\xi_{0}\right) \leq 0$, a contradiction. Therefore, $|f|^{\#} \equiv$ $\max \{f, 0\}$.

Proof of Theorem 1.1(b). Let $f=\left(\max \left\{f_{L}, 0\right\}\right)^{1 / n}$. Then $\left|f_{L}\right| \geq$ $\left|f_{L}\right|^{\#}=f^{n}$. Using Hölder's inequality in definition (1.3), it follows that, for $1 \leq p<n$,

$$
\left[\left(f^{p}\right)^{\#}\right]^{n / p} \leq g_{p} \leq\left|f_{L}\right|^{\#}=f^{n} .
$$

Since $f$ is a homogeneous function of degree 1 which vanishes exactly on the set $K_{L}$ and is also $L^{n}$-mean coercive in the sense defined in Yan $\&$ Zhou [17], it follows from [17, Theorem 2.1] that $\left(f^{p}\right)^{\#}(\xi)=0$ if and only if $\xi \in K_{L}$ for $p \geq n-\epsilon$ with some $\epsilon>0$, and therefore, by (2.2), $g_{p}(\xi)=0$ if and only if $\xi \in K_{L}$. This proves the result.

Proposition 2.3. Let $g \geq 0$ be a rank-one convex function. If $g(\xi)=$ 0 for all $\xi \in Z_{L}$ then $g(\xi)=0$ for all $\xi \in K_{L}$.

Proof. Let $\xi \in K_{L} \backslash Z_{L}$. Then $f_{L}(\xi)<0$. In a similar way as above, we have $f_{L}\left(\xi+t_{1} \eta\right)=f_{L}\left(\xi+t_{2} \eta\right)=0$ for some $t_{1}<0<t_{2}$ with a given rank-one matrix $\eta$. Thus $g\left(\xi+t_{i} \eta\right)=0$ for $i=1$, 2, which, by the rank-one convexity of $g$, implies $g(\xi)=0$ and proves the result.

Proof of Theorem 1.5. From definition of semiconvex hulls, to prove the theorem, it is sufficient to prove the equalities:

$Q C_{p}^{+}\left(Z_{L}\right)=Q C_{p}^{+}\left(K_{L}\right), R C_{p}^{+}\left(Z_{L}\right)=R C_{p}^{+}\left(K_{L}\right), P C_{p}^{+}\left(Z_{L}\right)=P C_{p}^{+}\left(K_{L}\right)$.

Since the functions in $Q C_{p}^{+}\left(Z_{L}\right), R C_{p}^{+}\left(Z_{L}\right)$, and $P C_{p}^{+}\left(Z_{L}\right)$ are all rankone convex, these equalities follow easily from Proposition 2.3. The proof is completed.

\section{The lamination hull and attainment ReSults}

Given any set $K \subset \mathbb{M}^{m \times n}$ and number $p \in[1, \infty]$, define $\beta_{p}(K)$ to be the set of matrices $\xi \in \mathbb{M}^{m \times n}$ such that there exists a map 
$u=u_{\xi} \in W^{1, p}\left(\Omega ; \mathbf{R}^{m}\right)$ satisfying

$$
D u(x) \in K \quad \text { a.e. } x \in \Omega,\left.\quad u\right|_{\partial \Omega}=\xi x .
$$

Note that Lemma 3.1 in Yan [14] shows that the set $\beta_{p}(K)$ is independent of the domain $\Omega$. From this definition, Theorem 1.2 is equivalent to the following

Theorem 3.1. $\beta_{p}\left(Z_{L}\right)=\mathbb{M}^{n \times n}$ for all $1 \leq p<\frac{n L}{L+1}$.

From this theorem, a similar argument as used for [14, Theorem 4.4] also derives the following shaper result; we refer to [14] for details.

Theorem 3.2. Let $n \geq 3, L \geq 1$ and $1 \leq p<\frac{n L}{L+1}$. Then, for any piece-wise affine map $\varphi \in W^{1, p}\left(\Omega ; \mathbf{R}^{n}\right)$ and $\epsilon>0$, there exists a map $u_{\epsilon} \in \varphi+W_{0}^{1, p}\left(\Omega ; \mathbf{R}^{n}\right)$ satisfying $D u_{\epsilon}(x) \in Z_{L}=\partial K_{L}$ a.e. in $\Omega$ and $\left\|u_{\epsilon}-\varphi\right\|_{L^{p}(\Omega)}<\epsilon$.

Let $S_{\alpha}$ be the set defined by (1.8) in the introduction and define a unbounded two-well set

$$
W_{L}=S_{\alpha_{1}} \cup J S_{\alpha_{2}}
$$

where $\alpha_{2}=-L^{-1}-1$ and $\alpha_{1}$ is either $L^{\frac{1}{n-1}}-1$ or $L^{-1}-1$.

Notice that Proposition 1.3 implies $W_{L} \subset Z_{L}$; therefore, Theorem 3.1 follows from an attainment result for set $W_{L}$, which is a direct corollary of Theorem 1.4.

Theorem 3.3. $\beta_{p}\left(W_{L}\right)=\mathbb{M}^{n \times n}$ for all $1 \leq p<\frac{n L}{L+1}$.

To prove Theorem 3.3, or the more general Theorem 1.4, we need some techniques in convex integration theory; we refer to $[10,11,14]$ for more references on this theory.

Definition 3.1. Let $\mathcal{L}_{j}(K)$ be defined for $j=0,1,2, \cdots$ inductively as follows: $\mathcal{L}_{0}(K)=K$ and, for $j=0,1, \cdots$,

$$
\mathcal{L}_{j+1}(K)=\left\{t \xi+(1-t) \eta \mid t \in[0,1], \xi, \eta \in \mathcal{L}_{j}(K), \operatorname{rank}(\xi-\eta) \leq 1\right\} .
$$

The lamination hull of set $K, \mathcal{L}(K)$, is then defined to be

$$
\mathcal{L}(K)=\cup_{j=0}^{\infty} \mathcal{L}_{j}(K) .
$$

The following useful attainment result has been given in Yan [14]; see also Yan [13]. 
Theorem 3.4 ([14, Theorem 3.2]). Let $K \subset \mathbb{M}^{m \times n}$ be a closed set and let $A \subset \beta_{p}(K)$ be a set satisfying

$$
c_{0}=\sup _{\xi \in A} \frac{1}{|\Omega|} \int_{\Omega}\left|D u_{\xi}(x)\right|^{p} d x<\infty,
$$

where $u_{\xi} \in W^{1, p}\left(\Omega ; \mathbf{R}^{n}\right)$ is some map satisfying (3.1). Suppose the lamination hull $B=\mathcal{L}(A)$ is open and bounded. Then $B \subset \beta_{p}(K)$.

Remarks. 1) It follows from [14, Lemma 3.1] and the remark following the proof of [14, Theorem 3.2] that, for any bounded domain $\Sigma \subset \mathbf{R}^{n}$ and any $\xi \in B=\mathcal{L}(A)$, there exists a map $u=u_{\xi} \in W^{1, p}\left(\Sigma ; \mathbf{R}^{m}\right)$ such that

$$
D u(x) \in K \quad \text { a.e. } x \in \Sigma,\left.\quad u\right|_{\partial \Sigma}=\xi x
$$

and

$$
\frac{1}{|\Sigma|} \int_{\Sigma}|D u(x)|^{p} d x \leq \max \left\{c_{0}, \sup _{\eta \in B}|\eta|^{p}\right\} .
$$

2) A closer look at the proof of [14, Theorem 3.2] also shows that the map $u=u_{\xi}$ satisfying (3.5), (3.6) above depends only on the family $\left\{u_{\xi} \mid \xi \in A\right\}$ in (3.4) and any fixed number $q \geq p$; in particular, the solution $u=u_{\xi}$ for any $\xi \in B$ can be made independent of power $p$ as long as $p<q$ for some $q<\infty$. This can be seen from the choice of sequence $\left\{\epsilon_{k}\right\}$ in the proof of $[14$, Theorem 3.2]. We notice that the construction of $u=u_{\xi}$ depends on power $p$ only through this sequence $\left\{\epsilon_{k}\right\}$ that is required to satisfy $\epsilon_{k} \rightarrow 0^{+}$and $\sum_{k} \epsilon_{k}^{1 / p}<\infty$. However, if $p<q$, we can fix such a sequence $\epsilon_{k}$ satisfying $\epsilon_{k} \rightarrow 0^{+}$and $\sum_{k} \epsilon_{k}^{1 / q}<$ $\infty$. Then the solution $u=u_{\xi}$ constructed there is seen only depending on this sequence $\left\{\epsilon_{k}\right\}$ and the family $\left\{u_{\xi} \mid \xi \in A\right\}$ given in (3.4). Note that the estimate (3.6) is independent of the sequence $\left\{\epsilon_{k}\right\}$.

The main theorem of this section is the following

Theorem 3.5. Let $K \subset \mathbb{M}^{m \times n}$ be a closed set and $q>1$ a number given. Suppose $A \subset \mathbb{M}^{m \times n}$ is a set such that for each $\xi \in A$ there exists a map $v=v_{\xi} \in W^{1, q)}\left(\mathbf{B} ; \mathbf{R}^{m}\right)$ satisfying $D v(x) \in K$ a.e. $x \in \mathbf{B}$ and $\left.v\right|_{\partial \mathbf{B}}=\xi x$, where $\mathbf{B}$ is the unit ball in $\mathbf{R}^{n}$, and suppose that

$$
C_{q}=\sup _{\xi \in A}\left[D v_{\xi}\right]_{q, \mathbf{B}}<\infty .
$$

If the lamination hull $B=\mathcal{L}(A)$ is open and bounded, then, for any bounded domain $\Omega \subset \mathbf{R}^{n}$ and any $\xi \in B=\mathcal{L}(A)$, there exists a map 
$u=u_{\xi} \in W^{1, q)}\left(\Omega ; \mathbf{R}^{m}\right)$ satisfying (3.1) and, therefore, $B=\mathcal{L}(A) \subset$ $\beta_{p}(K)$ for all $1 \leq p<q$.

Proof. Condition (3.7) implies that, for any $1 \leq p<q$,

$$
c_{p}=\sup _{\xi \in A} \frac{1}{|\mathbf{B}|} \int_{\mathbf{B}}\left|D v_{\xi}(x)\right|^{p} d x \leq \frac{C_{q}^{p}}{q-p}<\infty .
$$

From this, using Theorem 3.4 quoted above and its remarks, we obtain, for each given $\xi \in B=\mathcal{L}(A)$, a map $u=u_{\xi}$ depending only on the power $q$ and the family $\left\{v_{\xi} \mid \xi \in A\right\}$ in (3.7) that belongs to $W^{1, p}\left(\Omega ; \mathbf{R}^{n}\right)$ for all $1 \leq p<q$ and satisfies (3.1) and

$$
\frac{1}{|\Omega|} \int_{\Omega}|D u|^{p} d x \leq \max \left\{c_{p}, \sup _{\eta \in B}|\eta|^{p}\right\}
$$

Multiplying this estimate by $q-p$, taking the $1 / p$ power, and maximizing over $1 \leq p<q$ show that

$$
[D u]_{q, \Omega} \leq \max \left\{C_{q}, \gamma_{q} \sup _{\eta \in B}|\eta|\right\}<\infty
$$

where $\gamma_{q}=\sup _{1 \leq p \leq q}(q-p)^{1 / p}<\infty$ is a constant. This shows $u=u_{\xi} \in$ $W^{1, q)}\left(\Omega ; \mathbf{R}^{n}\right)$ satisfying (3.1) and completes the proof.

\section{Proof of Theorem 1.4}

In this final section, we apply Theorem 3.5 to prove the main attainment theorem, Theorem 1.4. To do so, as in Yan $[13,14]$, we further introduce certain subsets in $\mathbb{M}^{n \times n}$. Let $W_{L}$ be the set defined by (3.2). Given a number $\lambda>0$, define

$$
\begin{aligned}
& R(n)=\left\{\left.\xi \in \mathbb{M}^{n \times n}|| \xi\right|^{n}=|\operatorname{det} \xi|\right\} ; \\
& A_{\lambda}=\{\xi \in R(n)|| \xi \mid<\lambda\}, \quad \tilde{A}_{\lambda}=A_{\lambda} \backslash\{0\} ; \\
& B_{\lambda}=\left\{\xi \in \mathbb{M}^{n \times n}|| \xi \mid<\lambda\right\} .
\end{aligned}
$$

Proposition 4.1. $\mathcal{L}_{n}\left(\tilde{A}_{\lambda}\right)=B_{\lambda}$. Therefore $B_{\lambda}=\mathcal{L}\left(\tilde{A}_{\lambda}\right)$ is a bounded open set.

Proof. Let $\xi \in B_{\lambda}$. If $\xi \neq 0$ then the proof of Proposition 4.1 in [14] has essentially shown that $\xi \in \mathcal{L}_{n-1}\left(\tilde{A}_{\lambda}\right)$. Therefore one has only to show $0 \in \mathcal{L}_{n}\left(\tilde{A}_{\lambda}\right)$. To prove this, let $0<t<\lambda$ be fixed. Note that, since the diagonal matrices $\delta\left(\epsilon_{1}, \epsilon_{2}, \cdots, \epsilon_{n}\right)$ and $\delta\left(-\epsilon_{1}, \epsilon_{2}, \cdots, \epsilon_{n}\right)$, where $\epsilon_{i}=$ 
$\pm t$, are in $\tilde{A}_{\lambda}$ and their difference is rank-one, by Definition 3.1, we have

$$
\delta\left(0, \epsilon_{2}, \cdots, \epsilon_{n}\right)=\frac{\delta\left(\epsilon_{1}, \epsilon_{2}, \cdots, \epsilon_{n}\right)+\delta\left(-\epsilon_{1}, \epsilon_{2}, \cdots, \epsilon_{n}\right)}{2}
$$

belongs to $\mathcal{L}_{1}\left(\tilde{A}_{\lambda}\right)$ for all $\epsilon_{i}= \pm t$. Now that matrices $\delta\left(0, \epsilon_{2}, \cdots, \epsilon_{n}\right)$ and $\delta\left(0,-\epsilon_{2}, \cdots, \epsilon_{n}\right)$ are in $\mathcal{L}_{1}\left(\tilde{A}_{\lambda}\right)$ and differ by rank-one, hence

$$
\delta\left(0,0, \epsilon_{3}, \cdots, \epsilon_{n}\right)=\frac{\delta\left(0, \epsilon_{2}, \epsilon_{3}, \cdots, \epsilon_{n}\right)+\delta\left(0,-\epsilon_{2}, \epsilon_{3}, \cdots, \epsilon_{n}\right)}{2}
$$

is in $\mathcal{L}_{2}\left(\tilde{A}_{\lambda}\right)$ for all $\epsilon_{i}= \pm t$. Repeating this argument finitely many steps, we eventually arrive at $0=\delta(0,0, \cdots, 0) \in \mathcal{L}_{n}\left(\tilde{A}_{\lambda}\right)$.

Proposition 4.2. Let $\lambda>0, n \geq 3, L \geq 1$ and $q=\frac{n L}{L+1}$. Then, for each $\xi \in \tilde{A}_{\lambda}$, there exists a map $v=v_{\xi} \in W^{1, q)}\left(\mathbf{B} ; \mathbf{R}^{n}\right)$ satisfying $D v(x) \in W_{L}$ a.e. $x \in \mathbf{B}$ and $\left.v\right|_{\partial \mathbf{B}}=\xi x$, such that

$$
c_{0} \equiv \sup _{\xi \in \tilde{A}_{\lambda}}\left[D v_{\xi}\right]_{q, \mathbf{B}} \leq C(n, L) \lambda<\infty .
$$

Proof. Let $\xi \in \tilde{A}_{\lambda}$ be given. We look for map $v=v_{\xi}$ in the form of radial maps: $v(x)=|x|^{\alpha} \xi x$, where $\alpha$ is a number to be chosen later. Note that all such maps satisfy $\left.v\right|_{\partial \mathbf{B}}=\xi x$ in the $W^{1, p}$-sense if $v \in W^{1, p}\left(\mathbf{B} ; \mathbf{R}^{n}\right)$ for some $p \geq 1$. Let $r=|x|, \omega=r^{-1} x$; then a simple calculation shows that

$$
\begin{aligned}
& D v(x)=r^{\alpha} \xi(I+\alpha \omega \otimes \omega), \\
& \operatorname{det} D v(x)=(1+\alpha) r^{\alpha n} \operatorname{det} \xi \\
& |D v(x)|= \begin{cases}r^{\alpha}|\xi|, & |1+\alpha| \leq 1, \\
|1+\alpha| r^{\alpha}|\xi|, & |1+\alpha|>1 .\end{cases}
\end{aligned}
$$

If $\operatorname{det} \xi>0$, we choose $\alpha=\alpha_{1}$ to be one of the two numbers defined above, and then one easily sees that $D v(x) \in S_{\alpha_{1}}$ for all $x \neq 0$ in $\mathbf{B}$ and, a computation also shows that this function $v=v_{\xi}$ belongs to $W^{1, q)}\left(\mathbf{B} ; \mathbf{R}^{n}\right)$ and satisfies

$$
\left[D v_{\xi}\right]_{q, \mathbf{B}} \leq C_{1}(n, L)|\xi| .
$$

If $\operatorname{det} \xi<0$, we let $\alpha=\alpha_{2}=-L^{-1}-1$ and in this case one easily sees that $D v_{\xi}(x) \in J S_{\alpha_{2}}$ for all $x \neq 0$. Furthermore, we compute to get

$$
\frac{1}{|\mathbf{B}|} \int_{\mathbf{B}}\left|D v_{\xi}(x)\right|^{p} d x=|\xi|^{p} \int_{0}^{1} r^{\alpha_{2} p+n-1} d r=\frac{|\xi|^{p}}{\alpha_{2} p+n}=\frac{L}{L+1} \frac{|\xi|^{p}}{q-p}
$$


which shows that $v=v_{\xi}$ belongs to $W^{1, q)}\left(\mathbf{B} ; \mathbf{R}^{n}\right)$ and satisfies

$$
\left[D v_{\xi}\right]_{q, \mathbf{B}} \leq C_{2}(n, L)|\xi| \text {. }
$$

Combining (4.8), (4.9), one proves (4.4). The proof is completed.

Proof of Theorem 1.4. Let $\xi \in \mathbb{M}^{n \times n}$ be given. Let $\lambda>0$ be a number such that $|\xi|<\lambda$. Then $\xi \in B_{\lambda}$. From Proposition 4.1, $\mathcal{L}\left(\tilde{A}_{\lambda}\right)=B_{\lambda}$ is open and bounded. Also, from Proposition 4.2, the set $A=\tilde{A}_{\lambda}$ satisfies the condition (3.7) in Theorem 3.5 with $K=W_{L}$ and $q=\frac{n L}{L+1}$. Therefore, Theorem 3.5 implies that, there exists a map $u=u_{\xi} \in W^{1, q)}\left(\Omega ; \mathbf{R}^{n}\right)$ satisfying $D u(x) \in W_{L}$ for a.e. $x \in \Omega$ and $\left.u\right|_{\partial \Omega}=\xi x$. Note also that $[D u]_{q, \Omega} \leq C_{0} \lambda<\infty$. We complete the proof.

\section{REFERENCES}

[1] E. Acerbi and N. Fusco, Semicontinuity problems in the calculus of variations, Arch. Rational Mech. Anal., 86 (1984), 125-145.

[2] J. M. Ball, Convexity conditions and existence theorems in nonlinear elasticity, Arch. Rational Mech. Anal., 63 (1977), 337-403.

[3] J. M. Ball and R. D. James, Proposed experimental tests of a theory of fine microstructures and the two well problem, Phil. Trans. Roy. Soc. London, 338A (1992), 389-450.

[4] J. M. Ball and F. Murat, $W^{1, p}-$ Quasiconvexity and variational problems for multiple integrals, J. Funct. Anal., 58 (1984), 225-253.

[5] B. Dacorogna, "Direct Methods in the Calculus of Variations." SpringerVerlag; Berlin, 1989.

[6] T. Iwaniec, An approach to Cauchy-Riemann operators in $\mathbf{R}^{n}$, preprint.

[7] T. Iwaniec and C. Sbordone, On the integrability of the Jacobian under minimal hypotheses, Arch. Rational Mech. Anal., 119 (1992), 129-143.

[8] C. B. Morrey, "Multiple Integrals in the Calculus of Variations." SpringerVerlag; Berlin, 1966.

[9] S. Müller, Variational models for microstructure and phase transitions, in "Calculus of Variations and Geometric Evolution Problems," (Cetraro, 1996), pp. 85-210, Lecture Notes in Math. 1713, Springer, 1999.

[10] S. Müller and V. Šverák, Attainment results for the two-well problem by convex integration, Geometric analysis and the calculus of variations, 239251, Internat. Press; Cambridge, MA, 1996.

[11] V. Šverák, On the problem of two wells, in "Microstructure and Phase Transition," pp. 183-190, (D. Kinderlehrer et al eds.), Springer-Verlag; New York, 1993.

[12] B. Yan, On rank-one convex and polyconvex conformal energy functions with slow growth, Proc. Roy. Soc. Edinburgh, 127A (1997), 651-663.

[13] B. Yan, Linear boundary values of weakly quasiregular mappings, C. R. Acad. Sci. Paris Ser. I Math., 331 (2000), 379-384. 
[14] B. Yan, A linear boundary value problem for weakly quasiregular mappings, Cal. Var. Partial Differential Equations, to appear.

[15] B. Yan, Semiconvex hulls of quasiconformal sets, J. Convex Analysis, to appear.

[16] B. Yan and Z. Zhou, Stability of weakly almost conformal mappings, Proc. Amer. Math. Soc., 126 (1998), 481-489.

[17] B. Yan and Z. Zhou, $L^{p}$-mean coercivity, regularity and relaxation in the calculus of variations, Nonlinear Analysis, to appear.

[18] K. Zhang, On various semiconvex hulls in the calculus of variations, Cal. Var. Partial Differential Equations, 6 (1998), 143-160.

[19] K. Zhang, Rank-one connections at infinity and quasiconvex hulls, J. Convex Analysis, 7 (2000), 19-45.

Department of Mathematics, Michigan State University, East LansING, MI 48824, USA

E-mail address: yan@math.msu.edu 